\title{
Intensity of Insecticide Resistance in the Major Malaria Vector Anopheles Funestus From Chikwawa, Rural Southern Malawi
}

\section{Justin Kumala}

Wits University: University of the Witwatersrand

Lizette L Koekemoer

Wits University: University of the Witwatersrand

\section{Maureen Coetzee}

Wits University: University of the Witwatersrand

Themba Mzilahowa ( $\nabla$ tmzilahowa@mac.medcol.mw)

University of Malawi College of Medicine

\section{Research Article}

Keywords: Pyrethroids, susceptibility test, bioassay, insecticide-treated nets, indoor residual spraying, malaria transmission, vector control, sub-Saharan Africa

Posted Date: January 18th, 2022

DOI: https://doi.org/10.21203/rs.3.rs-1231906/v1

License: (a) (i) This work is licensed under a Creative Commons Attribution 4.0 International License. Read Full License 


\section{Abstract}

\section{Background}

Malaria vector control using insecticide-based approaches, has proven to be an effective strategy. However, widespread insecticide resistance among malaria vector populations across sub-Saharan Africa threatens to derail control efforts. This study was conducted in Chikwawa district, an area in rural southern Malawi characterised by persistent malaria transmission and reports of insecticide resistance in the local mosquito population. The study aimed to characterise the intensity of insecticide resistance within a population of Anopheles funestus, a major vector of malaria in this district.

\section{Methods}

Live adult females belonging to the An. funestus group were collected from households by indoor aspiration. A modified CDC bottle assay was used for phenotypic quantification of resistance to deltamethrin, permethrin and alpha-cypermethrin at $1 \mathrm{X}, 2.5 \mathrm{X}, 5 \mathrm{X}$ and $10 \mathrm{X}$ the recommended diagnostic doses. WHO tube assays were used to determine susceptibility to bendiocarb, dichlorodiphenyltrichloroethane (DDT) and pirimiphos-methyl insecticides at diagnostic concentrations.

\section{Results}

Anopheles funestus exposed to $10 x$ concentrations was highly resistant to alpha-cypermethrin (mortality $95.4 \%$ ) whereas there was $100 \%$ mortality to both deltamethrin and permethrin at the same dose. Despite susceptibility at 10X, mortality to deltamethrin and permethrin at $5 \mathrm{X}$ was $96.7 \%$ and $97.1 \%$ respectively, indicating moderate resistance to the two insecticides. WHO susceptibility assays indicated strong resistance against bendiocarb (mortality $33.8 \%$, exposed $n=93$ ) whereas there was full susceptibility to DDT (mortality $98.9 \%, n=103$ ) and pirimiphos-methyl (mortality $100 \%, n=103$ ).

\section{Conclusions}

Strategies for managing resistance, particularly against pyrethroids, must be urgently implemented to maintain the effectiveness of bed nets, which remain the primary vector control intervention in the area. This includes the wide scale introduction of third generation synergist ITNs and next-generation dual active ingredient ITNs. The use of effective non-pyrethroids such as pirimiphos-methyl, clothianidin and potentially DDT, could provide a window of opportunity for IRS across the district. This would support the current Malawi Insecticide Resistance Management Plan which aims at rotating insecticides to minimise selection pressure and slow down the evolution of resistance to approved insecticides. This will help to prevent malaria vector control failure and improve progress towards malaria elimination.

\section{Background}

There have been tremendous gains in reducing the global burden of malaria over the past two decades despite progress stalling in the last few years [1]. In the African region, it is estimated that Plasmodium 
falciparum prevalence declined by $50 \%$ while incidence decreased by $42 \%$ over the same period. In Malawi, the implementation of evidence-based policies including the provision of indoor residual spraying (IRS), insecticide treated bed nets (ITNs) and intermittent preventive therapy for pregnant women (iPTp) contributed to a decline in malaria prevalence from $44 \%$ in 2010 to $22 \%$ by 2017 [2]. Mean modelled prevalence rates of Plasmodium falciparum declined from 29\% in 2010 to $15 \%$ in 2017 with percent decline ranging from $3-79 \%$ in some areas [3]. However, these gains are threatened by the rise of insecticide resistance across malaria vector populations both locally and across sub-Saharan Africa [4-8].

In Malawi, resistance to at least one insecticide has been reported in many areas of the country, including multiple resistance in Chikwawa district. Chikwawa is an area along the Lower Shire Valley with persistent and one of the highest transmission risk areas with $P$. falciparum prevalence rates as high as $15 \%(n=9646)$ among participants in a recent study [9]. .Anopheles funestus s.s, the primary malaria vector in this district, has shown reduced susceptibility to pyrethroids and carbamates and this appears to be enzyme-mediated resistance [10-12]. Anopheles arabiensis, a secondary vector in the district has also shown reduced levels of susceptibility to pyrethroids $[10,11,13] .$. However, the extent to which the rise of insecticide resistance might be hampering malaria control efforts in the district remains underexplored. The current study was part of a larger study which aimed at investigating the impact of insecticide resistance on vector control efforts in the district.

The level of pyrethroid resistance in Malawian An. funestus has slowly risen since it was first reported in 2008 by Hunt et al. [14]. In 2015, reports from Chikwawa district indicated the rise of multiple resistance mechanisms in the local An. funestus population, namely over-expression of pyrethroid resistance genes (CYP6Pa/b and CYP7M7) and the dieldrin resistance mutation (A296S-RDL) [12, 15, 16]. Such reports suggest that insecticide-based vector control tools (such as ITNs and IRS) may slowly lose their efficacy especially in the wake of multiple resistance as recently reported in neighbouring Mozambique where the same resistance mechanisms in the local An. funestus population have induced a loss of efficacy of piperonyl-butoxide (PBO)-based ITNs [17], indicating other uncharacterized mechanisms at play. In Cote d'Ivoire, PBO pre-exposure of resistant An. gambiae s.I. did not yield full restoration of susceptibility to pyrethroids and neither was there full susceptibility to chlorfenapyr, a novel insecticide for public health used in combination with pyrethroids in the newer generation ITNs [18].

Malawi has a lack of compelling evidence as to what relationship exists between resistance and the effect of ITNs on mosquito mortality in the field. It is unknown if there is compromised transmission control for people using ITNs, and if there is any relationship between mosquito resistance and epidemiological profiles of clinical malaria in the district. These factors are important in establishing the real impact of resistance to operational malaria control [19-21]. This study focused on determining the intensity of resistance to deltamethrin, permethrin and alpha-cypermethrin (pyrethroids) and alternative non-pyrethroid insecticides. This was done so that an operationally important level of resistance could be determined that may give an early warning signal of potential loss of efficacy in order to guide the local vector control intervention policy and minimise or prevent control failure. 


\section{Methods}

\section{Study Design}

This entomological study was conducted after the rainy season, between April and July 2018 across two

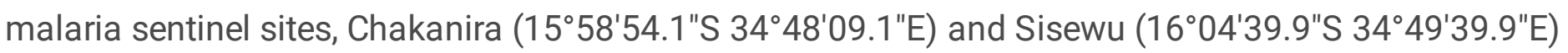
villages in Chikwawa district, southern Malawi. Malaria cases peak just after the rainy season which lasts from December to February.

\section{Mosquito collections}

Collections of live adult mosquitoes were carried out inside houses using a battery powered Prokopak Aspirator. Mosquitoes were placed in paper cups which were put in cooler boxes and provided with a sugar solution prior to transport to the laboratory in Blantyre. The local vectors are Anopheles funestus (most abundant) and Anopheles arabiensis [22].

\section{CDC bottle assays}

Tests were performed on three replicates of 20-25 wild-caught adult females of unknown age which were exposed for 1 hour in $250 \mathrm{~mL}$ glass bottles coated with insecticide concentrations at 1X diagnostic concentration (DC), 2.5X DC, 5X DC and 10X DC of pyrethroids (permethrin, deltamethrin and alphacypermethrin). Negative controls were bottles coated with acetone, a solvent used for dilution of the test insecticides. Knockdown was recorded at 10-minute intervals from the time of exposure until 1 hour, when mosquitoes were then transferred to holding cages. Mortality was recorded 24 hours from exposure. A small piece of cotton wool moistened with $10 \%$ sugar solution was provided to survivors [23].

\section{WHO Susceptibility tests}

Wild-caught live female Anopheles funestus mosquitoes were tested for phenotypic resistance to DDT $(4 \%)$, bendiocarb $(0.1 \%)$ and pirimiphos-methyl $(0.25 \%)$ using standard WHO insecticide susceptibility assays [23]. Mosquitoes were exposed to treated papers for one hour and then transferred to holding tubes and provided with $10 \%$ sugar solution. Knockdown was assessed every 10 minutes from exposure to 60 minutes after exposure, and mortality was recorded at 24 hours.

\section{Species Identification by Polymerase Chain Reaction}

Following bioassay exposures, a sample of the An. funestus population was subjected to species identification using polymerase chain reaction (PCR). Samples were analysed using standard operating procedures for identifying An. funestus group members Koekemoer, Kamau [24]Cohuet, Simard [25] for identifying members of the An. funestus group. The PCR conditions were as follows: 1 cycle at $94^{\circ} \mathrm{C}$ for 2 minutes, 30 cycles at $94^{\circ} \mathrm{C}$ for 30 seconds, $45^{\circ} \mathrm{C}$ for 30 seconds, $72^{\circ} \mathrm{C}$ for 40 seconds and finally 1 cycle at $72^{\circ} \mathrm{C}$ for 5 minutes.

\section{Results}


A total of 1,332 live wild An. funestus s.l. females were collected, of which 1,153 came from Chakanira village while 179 were from Sisewu village. These were used for insecticide susceptibility tests (CDC bottle assays and WHO tube assays). A subsample of 400 (75 from Sisewu and 325 from Chakanira) was then selected for molecular identification. A small number of An. gambiae s./ was also collected (1specimen from Chakanira and 12 from Sisewu) but these were not processed further.

\section{CDC Bottle Assays}

Table 1 summarizes the results of the pyrethroid intensity tests for the two villages, giving the numbers of mosquitoes tested per insecticide concentration (dose), percent mortality rates, and adjusted mortality rates using Abbott's formula [23]. 
Table 1

CDC bottle bioassays of An. funestus s.I. for Chakanira and Sisewu villages

\begin{tabular}{|c|c|c|c|c|c|}
\hline Village & Insecticide & Dose & $\begin{array}{l}\text { No. } \\
\text { exposed } \#\end{array}$ & $\begin{array}{l}\text { Unadjusted \% } \\
\text { Mortality }\end{array}$ & $\begin{array}{l}\text { Adjusted \% } \\
\text { Mortality* }\end{array}$ \\
\hline \multirow[t]{13}{*}{ Chakanira } & Control & $0 X$ & 133 & 9.0 & 0.0 \\
\hline & Deltamethrin & $1 X$ & 38 & 84.2 & 82.6 \\
\hline & & $2.5 X$ & 90 & 82.2 & 80.5 \\
\hline & & $5 X$ & 75 & 94.7 & 94.1 \\
\hline & & $10 \mathrm{X}$ & 71 & 100.0 & 100.0 \\
\hline & Permethrin & $1 X$ & 73 & 93.2 & 92.5 \\
\hline & & $2.5 \mathrm{X}$ & 62 & 93.5 & 92.9 \\
\hline & & $5 x$ & 68 & 97.1 & 96.8 \\
\hline & & $10 x$ & 77 & 100.0 & 100.0 \\
\hline & $\begin{array}{l}\text { Alpha- } \\
\text { cypermethrin }\end{array}$ & $1 X$ & 52 & 84.6 & 83.1 \\
\hline & & $2.5 X$ & 53 & 86.8 & 85.5 \\
\hline & & $5 X$ & 74 & 95.9 & 95.5 \\
\hline & & $10 x$ & 65 & 95.4 & 94.9 \\
\hline \multirow[t]{5}{*}{ Sisewu§ } & Control & $0 X$ & 25 & 8.0 & 0.0 \\
\hline & Deltamethrin & $1 X$ & 21 & 90.5 & 89.6 \\
\hline & & $2.5 X$ & 25 & 92.0 & 91.3 \\
\hline & & $5 X$ & 38 & 94.7 & 94.3 \\
\hline & & $10 \mathrm{x}$ & 17 & 100.0 & 100.0 \\
\hline \multicolumn{6}{|c|}{ *Adjusted mortality using Abbott's formula as described by WHO, 2016} \\
\hline \multicolumn{6}{|c|}{ \#Low sample numbers could have affected these tests } \\
\hline
\end{tabular}

Figures 2 - 4 show the percentage knockdown of An. funestus s.l. from Chakanira village to the four concentrations of the three pyrethroids. Mortalities were adjusted using Abbot's correction formula as per the WHO test procedures [23]. 


\section{Resistance Intensity}

The strength of resistance also called intensity, was characterised for the three pyrethroids by comparing the mortality at 24 hours for each of the insecticides at various multiples of the diagnostic dose (Table 1). Resistance intensity is herein classified as low, moderate or high using a threshold of $90 \%$ for determining a resistant population at diagnostic dose (1X DC) [26, 27]. Observed mortalities at the diagnostic dose (1X DC) were $82.6 \%(n=38)$ for deltamethrin, $92.5 \%(n=73)$ for permethrin and $83.1 \%(n=52)$ for alphacypermethrin indicating confirmed resistance to both deltamethrin and alpha-cypermethrin, and suspected resistance to permethrin (Table 1, Fig. 5). For deltamethrin and alpha-cypermethrin, mortalities were still under the $90 \%$ threshold at 2.5X DC while they were in the range $90-98 \%$ at 5X DC indicating the presence of moderate resistance intensity in the Chakanira population [27].

An analysis of variance (ANOVA) was done to check for differences or similarities between mean mortalities across the insecticides at the various doses (1X DC, 2.5X DC etc). The Tukey's post-hoc test was used for pairwise comparisons of group means at a confidence level of 95\% (Figure 6).

For the given doses, ANOVA test results were as follows: $1 X D C, d f=2, F=0.71, p=0.543 ; 2.5 X D C, d f=2$, $F=0.49, p=0.636 ; 5 X$ DC, $d f=2, F=1.83, p=0.240 ; 10 X \quad D C, d f=2, F=4.77, p=0.087$. The ANOVA test assumed equal variances for the analysis, with a null hypothesis of "all means were equal" against the alternative "not all means were equal" with a significance level set at $5 \%$. The resulting ANOVA test probabilities were all higher than the set significance level (alpha $=0.05)$. This indicated that the test could not find an instance where the group mean for each insecticide at a given dose, was actually statistically significantly different from the others. The F-test failed to determine that the variability in the mean mortalities of the groups was sufficiently larger than the variability observed within the groups, as indicated by the small ratios and large probabilities ( $p$-value greater than 0.05 ). In other words, statistically speaking, the mean mortalities between the insecticides at each given dose belonged to the same population (were similar) and there was no evidence that any one of the insecticides performed better than the other at a given dose. This is also indicated in the interval plots by the overlapping $95 \%$ confidence intervals of the means (Fig. 7).

\section{WHO Susceptibility tests}

From the total live collections, 408 specimens were used for WHO susceptibility tests against DDT, bendiocarb, pirimiphos methyl and permethrin. Table 2 summarises An. funestus s.l. numbers tested for Chakanira and Sisewu villages. Only a single WHO susceptibility test was done for Sisewu village on permethrin, due to low numbers of mosquitoes collected. 
Table 2

Mosquito susceptibility to non-pyrethroids for Chakanira village

\begin{tabular}{|lllll|}
\hline Village & Insecticide & Dose & No. exposed & \% Mortality \\
\hline \multirow{3}{*}{ Chakanira } & Control & OX & 29 & 0 \\
\cline { 2 - 5 } & DDT & 1X & 103 & 98.9 \\
\cline { 2 - 5 } & Control & OX & 27 & 0 \\
\cline { 2 - 5 } & Bendiocarb & 1X & 93 & 33.8 \\
\cline { 2 - 5 } & Pirimiphos-methyl & 1X & 103 & 100 \\
\hline \multirow{2}{*}{ Sisewu } & Control & OX & 28 & 0.0 \\
\cline { 2 - 5 } & Permethrin & 1X & 25 & 35.4 \\
\cline { 2 - 5 } & & &
\end{tabular}

\section{Molecular Species Identification}

The results for species identification of 400 wild females revealed that $A n$. funestus s.s. was the most abundant member of the An. funestus group $(\mathrm{n}=392)$ followed by An. parensis $(\mathrm{n}=6)$ and An. rivulorum $(n=2)$. While the majority of these specimens were from Chakanira village, 75 of them were from Sisewu village and identified as follows: 72 An. funestus s.s., 2 An. rivulorum and 1 An. parensis.

\section{Discussion}

This study highlighted high resistance intensity to alpha cypermethrin and moderate resistance to deltamethrin and permethrin in An. funestus s.I. from Chikwawa. Anopheles funestus s.l. exposed to deltamethrin indicated confirmed resistance at 1X DC, and when assayed at 2.5X DC and 5X DC, the mortality was less than $98 \%$. Susceptibility was only restored when a $10 \mathrm{X}$ dose was used, hence the classification as moderate intensity [27]. Similar results were observed for permethrin with the only difference being that mortality at 1 X DC was $92.5 \%$ indicating possible resistance, which was confirmed when higher doses were used at 2.5X and $5 \mathrm{X}$ DC with mortalities ranging from $90-98 \%$. Susceptibility was also restored at 10X DC as with deltamethrin, indicating moderate resistance. Mortality to alphacypermethrin was less than $90 \%$ at 1 X DC and $2.5 X$ DC, while it fell in the range $90-98 \%$ at $5 \mathrm{X}$ and $10 \mathrm{X}$ $\mathrm{DC}$, indicative of high intensity of resistance to this pyrethroid.

In addition to resistance against all the three pyrethroids, low mortality $(33.8 \%, \mathrm{n}=93)$ of An. funestus to the carbamate bendiocarb was also observed. However, intensity assays were not carried out in this study and this warrants further investigations. Previous studies in southern Malawi reported that An. funestus mortality to bendiocarb dropped from $60 \%$ in 2009 to $30.1 \%$ in 2014 , before a further decrease to $19 \%$ in 2015 [15]. Unlike previous studies which reported moderate resistance or variable responses to DDT in the An. funestus population [12,15], complete susceptibility to DDT (mortality $98.9 \%, n=103$ ) was observed in 
the current study. The current study also corroborates previous observations of full susceptibility to organophosphates with observed mortality of $100 \%(n=103)$ to pirimiphos-methyl $[10-13,15]$.

This study used wild-caught female Anopheles funestus group mosquitoes of unknown age and did not rear any specimens in the laboratory or insectary for the insecticide susceptibility tests. One limitation of using wild mosquitoes was the fact they could have been exposed to various field conditions including varying degrees of insecticide doses (e.g. on nets, wall surfaces or in agricultural fields), therefore potentially running the risk of over-estimating resistance in the given mosquito population [23]. However, the given scenario more closely represents the actual conditions that any given intervention would encounter in the field, in addition to the fact that wild-collected specimen are also the epidemiologically relevant cohort of vectors [23]. Another limitation of the current study was that we could not test many insecticides at all doses due to low sample numbers. However, even given the low numbers, the study was still able to document the problem and its severity. Advanced molecular or biochemical tests for resistance mechanisms were not done as these were previously described in the study area by Riveron et al. [15].

The Malawi National Malaria Control Program (NMCP) recently adopted an Integrated Vector Control Strategy (IVCS) 2020-2024 and the Insecticide Resistance Management Plan (IRMP) 2019-2022 which currently form the core of the malaria control program in the country. For vector control, universal coverage of all at-risk populations with insecticide-treated nets is the main approach together with complementary indoor residual spraying in selected districts. ITNs are distributed via mass campaigns every three years and through routine distribution in clinics to pregnant mothers and children under the age of five while IRS is implemented yearly in four districts $[10,28]$. However, the implementation of IRS over the years has not been consistent in all the selected districts due to logistical and financial constraints and the rise in pyrethroid resistance. The rise of pyrethroid resistance prompted the development of the strategic plan for monitoring and managing resistance (the IRMP) to counter the potential detrimental impact of resistance $[10,29]$. The current study demonstrates the importance of the IRMP to safeguard progress in malaria control.

Recently, the NMCP scaled down the IRS spraying to only one district (Nkhotakota) and switched to pirimiphos-methyl for two consecutive spray rounds, spaced one year apart but the program has bounced back to four districts for IRS. The insecticides of choice in the new IRS strategy include pirimiphos-methyl (organophosphate), clothianidin (neonicotinoid) and a formulation that combines clothianidin and deltamethrin, while the upscaling of IRS to more districts is also affected by high costs of the recommended alternative insecticides $[10,13]$.

Although DDT is still effective against local mosquitoes and is recommended in the current vector control strategy, the potential use of this insecticide is hampered by concerns surrounding its use [30-33]. In 2006, the WHO made a call for the use of DDT for vector control and provided clear and strict guidelines which it adopted from the Stockholm Convention, stipulating how countries can incorporate DDT for malaria control while strongly mitigating against any potential risks. In fact, following the 2006 WHO 
recommendation for the use of DDT in highly endemic settings, Malawi announced that it would pilot a DDT IRS program [32,34]. Evidence shows that countries that continue both timely and correct use of DDT for IRS can reduce malaria transmission by up to $90 \%$ [35]. When South Africa reintroduced DDT spraying in 2000 , they reduced the number of malaria cases and deaths drastically, managed to keep levels under control and by 2012, set the goal to eliminate the disease entirely [36,37]. The number of countries in Africa adopting DDT spraying for malaria control has risen, and since 2005, it includes Malawi's eastern and southern neighbour Mozambique, where An. funestus is a key vector too [38].

However, it has been argued that because Malawi is a largely agricultural economy, the benefits of using DDT for the fight against malaria may be outweighed by the potential detrimental effects on the agricultural industry and the economy at large [39]. This debate is also common in other African countries and some studies have even suggested that the potential benefits of DDT use for malaria vector control would be in the same order of magnitude as its detrimental effects on infant mortality [40]. Compounds such as clothianidin and deltamethrin/clothianidin combinations thus offer a good option for the rotation strategy with organophosphates for the IRS program. The current vector control strategy also recommends PBO-nets and combination ITNs for areas with high levels of pyrethroid resistance [10, 29]. The present study supports and highlights the critical importance of this strategy as resistance intensity data add predictive value for making informed insecticide choices, to prevent compromised transmission control $[26,27,41]$.

Clearly, a vector control strategy such as IRS that incorporates DDT and pirimiphos-methyl or other organophosphates may be a preferred means to managing the current situation of resistance. This would serve as an interim solution as novel compounds are being developed or await registration and WHO approval and recommendation. Also, strategies that incorporate insecticides with new generation modes of action, or those that do not involve the use of insecticides at all, should complement ongoing interventions to avoid further risks that resistance may pose to the control program. These findings also highlight the importance of making new generation ITNs (such as PBO-Nets) readily and widely accessible to communities as they have demonstrated to have greater entomological and epidemiological impact than standard LLINs in settings of high insecticide resistance [41]. Such a multipronged approach will aid the country as it transitions towards elimination.

In this study, Anopheles funestus s.s. was found to be the most abundant species, as also previously observed [11,12]. Anopheles parensis and An. rivulorum were detected in this study and continuous surveillance and monitoring of these species needs to be incorporated as a routine, as these two species have been shown elsewhere to be contributing to transmission and also found biting humans indoors [42, 43]. Very few An. gambiae s.I. were collected $(n=13)$ in this study and no further investigations were done.

\section{Conclusion}

In conclusion, the level of resistance in Chikwawa has grown over the years and the current intensity warrants an urgent solution. It highlights the importance of a resistance management strategy to cushion 
against the potential negative impact of resistance. More research needs to be done to further validate the intensity of resistance in An. arabiensis in this geographical region and to explore the intensity of resistance to the carbamate, bendiocarb.

\section{Declarations}

\section{Acknowledgements}

We would like to thank all study participants, Health Surveillance Assistants (HSAs), village chiefs and all people who supported us during the study. We thank the Wits Research Institute for Malaria, Centre for Emerging Zoonotic \& Parasitic Diseases at the National Institute for Communicable Diseases, and MAC Communicable Diseases Action Center for institutional support.

\section{Funding}

This research was supported by the Consortium for Advanced Research Training in Africa (CARTA). CARTA is jointly led by the African Population and Health Research Center and the University of the Witwatersrand and funded by the Carnegie Corporation of New York (Grant No--B 8606.R02), Sida (Grant No:54100029), the DELTAS Africa Initiative (Grant No: 107768/Z/15/Z). The statements made and views expressed are solely the responsibility of the researchers. LLK is supported by the DST/NRF South African Research Chairs Initiative (grant no. 64763).

\section{Availability of data and materials}

Datasets used for the analysis of findings of this study are available upon request via the authors' email addresses.

\section{Authors' Contributions}

JK, MC, and TM designed the study. JK conducted the field collection of specimen and bioassays. LK led the molecular lab work, JK drafted the original manuscript. MC, TM and LK led the interpretation of findings. All authors revised the manuscript.

\section{Ethics approval and consent to participate}

Ethical clearance for the study was obtained from the Human Research Ethics Committee at the University of the Witwatersrand in South Africa clearance number M170662, and from College of Medicine Research Ethics Committee (CoMREC) in Malawi clearance number P.12/17/2324. Study participants were recruited as volunteers from the study area and informed consent was obtained before participation.

\section{Consent for publication}

All authors read and approved the final manuscript, and consented for publication. 


\section{Competing interests}

The authors declare that they have no competing interests.

\section{References}

1. WHO, World Malaria Report 2020: 20 years of global progress and challenges. 2020; World Health Organization, Geneva, Switzerland.

2. Mwendera CA, De Jager C, Longwe H, Kumwenda S, Hongoro C, Phiri K, et al. Challenges to the implementation of malaria policies in Malawi. BMC Health Serv Res. 2019;19:194.

3. Chipeta MG, Giorgi E, Mategula D, Macharia PM, Ligomba C, Munyenyembe A, et al. Geostatistical analysis of Malawi's changing malaria transmission from 2010 to 2017. Wellcome Open Res. 2019;4:57.

4. Tizifa TA, Kabaghe AN, McCann RS, Van den Berg H, Van Vugt M, Phiri KS. Prevention Efforts for Malaria. Curr Trop Med Rep. 2018;5(1):41-50.

5. Choi KS, Christian R, Nardini L, Wood OR, Agubuzo E, Muleba M, et al. Insecticide resistance and role in malaria transmission of Anopheles funestus populations from Zambia and Zimbabwe. Parasit Vectors. 2014;7:464.

6. Moyes CL, Athinya DK, Seethaler T, Battle KE, Sinka M, Hadi MP, et al. Evaluating insecticide resistance across African districts to aid malaria control decisions. Proc Natl Acad Sci USA. 2020;117(36):22042-22050.

7. Owuor KO, Machani MG, Mukabana WR, Munga SO, Yan G, Ochomo E, et al. Insecticide resistance status of indoor and outdoor resting malaria vectors in a highland and lowland site in Western Kenya. PLoS One. 2021;16(3):e0240771.

8. Pinda PG, Eichenberger C, Ngowo HS, Msaky DS, Abbasi S, Kihonda J, et al. Comparative assessment of insecticide resistance phenotypes in two major malaria vectors, Anopheles funestus and Anopheles arabiensis in south-eastern Tanzania. Malar J. 2020;19:408.

9. Andronescu LR, Buchwald AG, Coalson JE, Cohee L, Bauleni A, Walldorf JA, et al. Net age, but not integrity, may be associated with decreased protection against Plasmodium falciparum infection in southern Malawi. Malar J. 2019;18:329.

10. Abeku T, et al. Insecticide Resistance Management Plan, Malawi, 2019 - 2022. 2019; Malaria Consortium.

11. PMI-VectorLink-Project, Malawi Entomological Monitoring Annual Report. 2019; Malaria Alert Centre.

12. Mzilahowa T, Chiumia M, Mbewe RB, Uzalili VT, Luka-Banda M, Kutengule A, et al. Increasing insecticide resistance in Anopheles funestus and Anopheles arabiensis in Malawi, 2011-2015. Malar J. 2016;15:563.

13. Abeku T, et al. Integrated Vector Control Strategy for Malaria Control, Malawi, 2020-2024. 2020; Malaria Consortium. 
14. Hunt R, Edwardes M, Coetzee M. Pyrethroid resistance in southern African Anopheles funestus extends to Likoma Island in Lake Malawi. Parasit Vectors. 2010;3:122.

15. Riveron JM, Chiumia M, Menze BD, Barnes KG, Irving H, Ibrahim SS, et al. Rise of multiple insecticide resistance in Anopheles funestus in Malawi: a major concern for malaria vector control. Malar J. $2015 ; 14: 344$.

16. Riveron JM, Ibrahim SS, Chanda E, Mzilahowa T, Cuamba N, Irving H, et al. The highly polymorphic CYP6M7 cytochrome P450 gene partners with the directionally selected CYP6P9a and CYP6P9b genes to expand the pyrethroid resistance front in the malaria vector Anopheles funestus in Africa. BMC Genomics. 2014;15:817.

17. Riveron JM, Huijben S, Tchapga W, Tchouakui M, Wondji MJ, Tchoupo M, et al. Escalation of pyrethroid resistance in the malaria vector Anopheles funestus induces a loss of efficacy of piperonyl butoxide-based insecticide-treated nets in Mozambique. J Infect Dis. 2019;220(3):467-475.

18. Kouassi BL, Edi C, Tia E, Konan LY, Akre MA, Koffi AA, et al. Susceptibility of Anopheles gambiae from Côte d'Ivoire to insecticides used on insecticide-treated nets: evaluating the additional entomological impact of piperonyl butoxide and chlorfenapyr. Malar J. 2020;19:454.

19. Kleinschmidt I, Bradley J, Knox TB, Mnzava AP, Kafy HT, Mbogo C, et al. Implications of insecticide resistance for malaria vector control with long-lasting insecticidal nets: a WHO-coordinated, prospective, international, observational cohort study. Lancet Infect Dis. 2018;18(6):640-649.

20. Kleinschmidt I, Mnzava AP, Kafy HT, Mbogo C, Bashir Al, Bigoga J, et al. Design of a study to determine the impact of insecticide resistance on malaria vector control: a multi-country investigation. Malar J. 2015;14:282.

21. Wondji CS, Coleman M, Kleinschmidt I, Mzilahowa T, Irving H, Ndula M, et al. Impact of pyrethroid resistance on operational malaria control in Malawi. Proc Natl Acad Sci USA. 2012;109(47):1906319070.

22. Mzilahowa T, Hastings IM, Molyneux ME, McCall PJ. Entomological indices of malaria transmission in Chikhwawa district, Southern Malawi. Malar J. 2012;11:380.

23. WHO, Test procedures for insecticide resistance monitoring in malaria vector mosquitoes. 2016; World Health Organization, Geneva, Switzerland.

24. Koekemoer L, Kamau L, Hunt RH, Coetzee M. A cocktail polymerase chain reaction assay to identify members of the Anopheles funestus (Diptera: culicidae) group. Am J Trop Med Hyg. 2002;66(6):804811.

25. Cohuet A, Simard F, Toto JC, Kengne P, Coetzee M, Fontenille D. Species identification within the Anopheles funestus group of malaria vectors in Cameroon and evidence for a new species. Am $J$ Trop Med Hyg. 2003;69(2):200-205.

26. Bagi J, Grisales N, Corkill R, Morgan JC, N'Fale S, Brogdon WG, et al. When a discriminating dose assay is not enough: measuring the intensity of insecticide resistance in malaria vectors. Malar $\mathrm{J}$. $2015 ; 14: 210$. 
27. Venter N, Oliver SV, Muleba M, Davies C, Hunt RH, Koekemoer LL, et al. Benchmarking insecticide resistance intensity bioassays for Anopheles malaria vector species against resistance phenotypes of known epidemiological significance. Parasit Vectors. 2017;10:198.

28. Ye Y, Andrada A. Estimating malaria incidence through modeling is a good academic exercise, but how practical is it in high-burden settings? Am J Trop Med Hyg. 2020;102(4):701-702.

29. WHO, Insecticide-treated nets for malaria transmission control in areas with insecticide-resistant mosquito populations: preferred product characteristics. 2021; World Health Organization, Geneva, Switzerland.

30. News24. Malawian MPs in bizzare debate over use of DDT to fight malaria. News24 2010; accessed 31st July 2021 from: https://www.news24.com/news24/Archives/City-Press/Malawian-MPs-inbizarre-debate-over-use-of-DDT-to-fight-malaria-20150430.

31. Kabange SC. African Countries Debate Using DDT in Anti-Malaria Efforts. 2011; accessed 31 July 2021 from: https://www.voanews.com/africa/african-countries-debate-using-ddt-anti-malariaefforts.

32. Van den Berg H. Global status of DDT and its alternatives for use in vector control to prevent disease. Environ Health Perspect. 2009;117(11):1656-1663.

33. Coetzee M, Koekemoer LL. Molecular systematics and insecticide resistance in the major African malaria vector Anopheles funestus. Ann Rev Entomol. 2013;58(1):393-412.

34. The-Lancet. DDT for malaria control: the issue of trade. Lancet. 2007;369(9558):248.

35. Rehwagen C. WHO recommends DDT to control malaria. BMJ (Clinical research ed.). 2006;333(7569):622.

36. Hargreaves K, Koekemoer LL, Brooke BD, Hunt RH, Mthembu J, Coetzee M. Anopheles funestus resistant to pyrethroid insecticides in South Africa. Med Vet Entomol. 2000;14(2):181-189.

37. Maharaj R, Morris N, Seocharan I, Kruger P, Moonasar D, Mabuza A, et al. The feasibility of malaria elimination in South Africa. Malar J. 2012;11:423.

38. Coleman M, Casimiro S, Hemingway J, Sharp B. Operational impact of DDT reintroduction for malaria control on Anopheles arabiensis in Mozambique. J Med Entomol. 2008;45(5):885-890.

39. Chanda E, Mzilahowa T, Chipwanya J, Mulenga S, Ali D, Troell P, et al. Preventing malaria transmission by indoor residual spraying in Malawi: grappling with the challenge of uncertain sustainability. Malar J. 2015;14:254.

40. Chen A, Rogan WJ. Nonmalarial Infant Deaths and DDT Use for Malaria Control. Emerg Infect Dis. 2003;9(8):960-964.

41. Gleave K, Lissenden N, Chaplin M, Choi L, Ranson H. Piperonyl butoxide (PBO) combined with pyrethroids in insecticide-treated nets to prevent malaria in Africa. Cochrane Database Syst Rev. 2021;5(5):CD012776.

42. Burke A, Dahan-Moss Y, Duncan F, Qwabe B, Coetzee M, Koekemoer L, et al. Anopheles parensis contributes to residual malaria transmission in South Africa. Malar J. 2019;18:257. 
43. Kawada H, Dida GO, Sonye G, Njenga SM, Mwandawiro C, Minakawa N. Reconsideration of Anopheles rivulorum as a vector of Plasmodium falciparum in western Kenya: some evidence from biting time, blood preference, sporozoite positive rate, and pyrethroid resistance. Parasit Vectors. 2012;5:230.

\section{Figures}

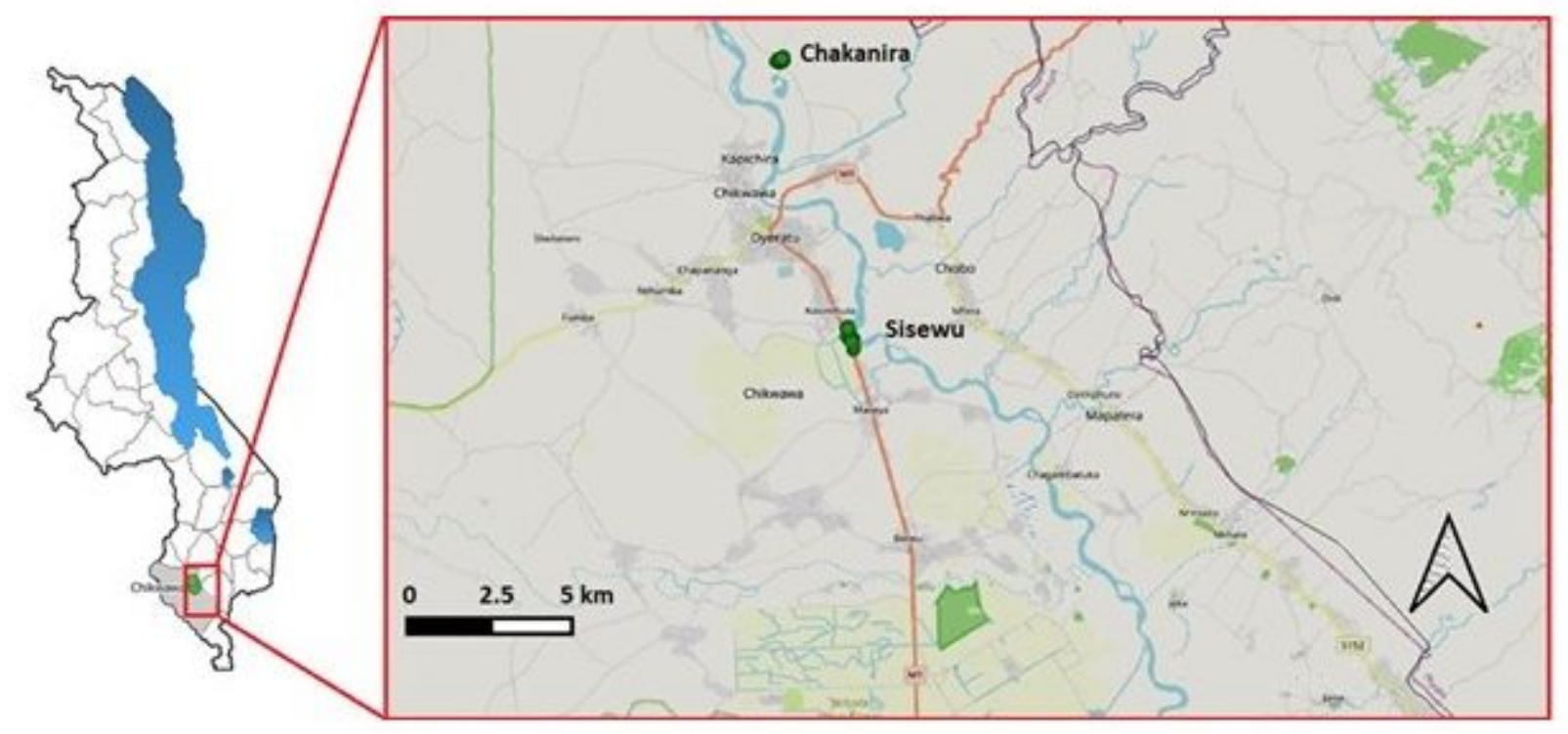

\section{Figure 1}

Map of Malawi, showing the location of Chikwawa district and study sites 


\section{Mortality of wild-caught An. funestus exposed to Deltamethrin}

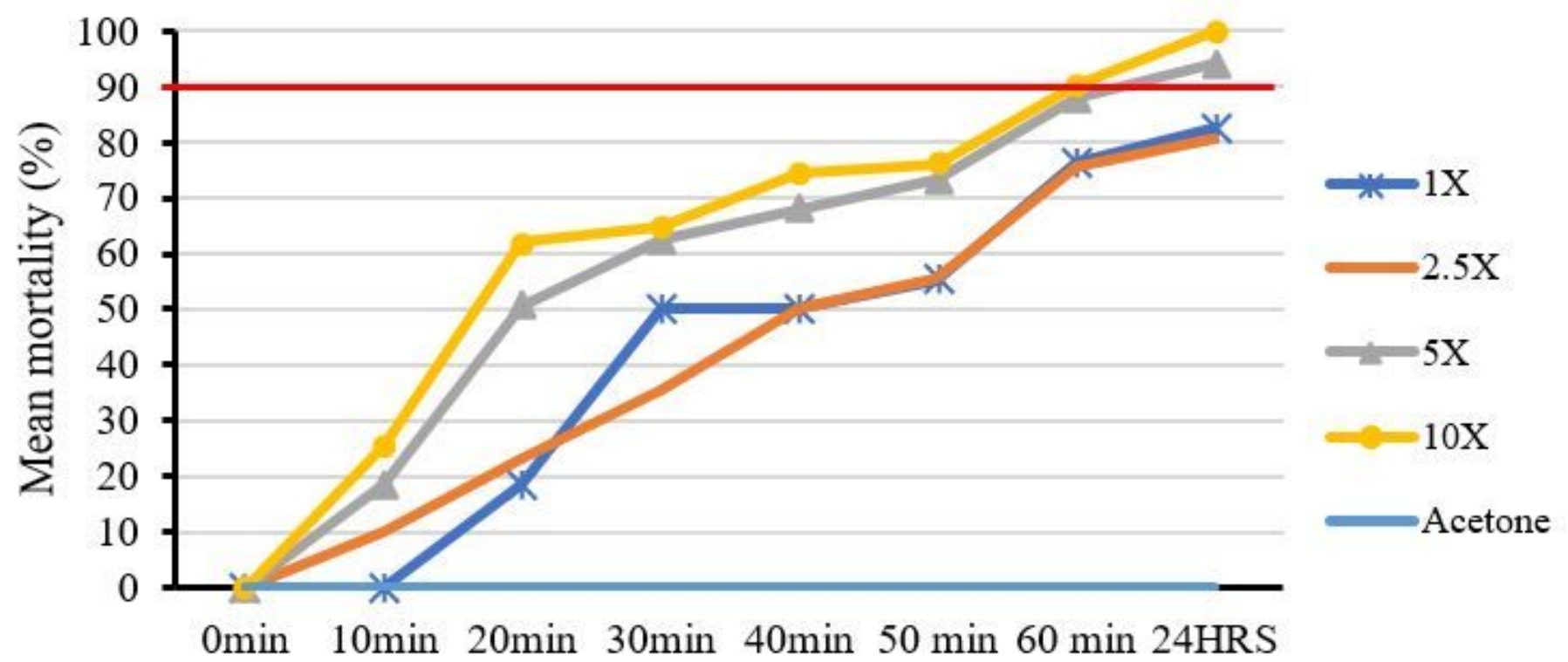

Time to knock down

\section{Figure 2}

Knockdown time and 24-hr mortality rates of wild-caught An. funestus s.l., after exposure to various concentrations of deltamethrin. The horizontal redline represents the $90 \%$ mortality threshold for confirmation of resistance 
Mortality of wild-caught An. funestus exposed to Permethrin

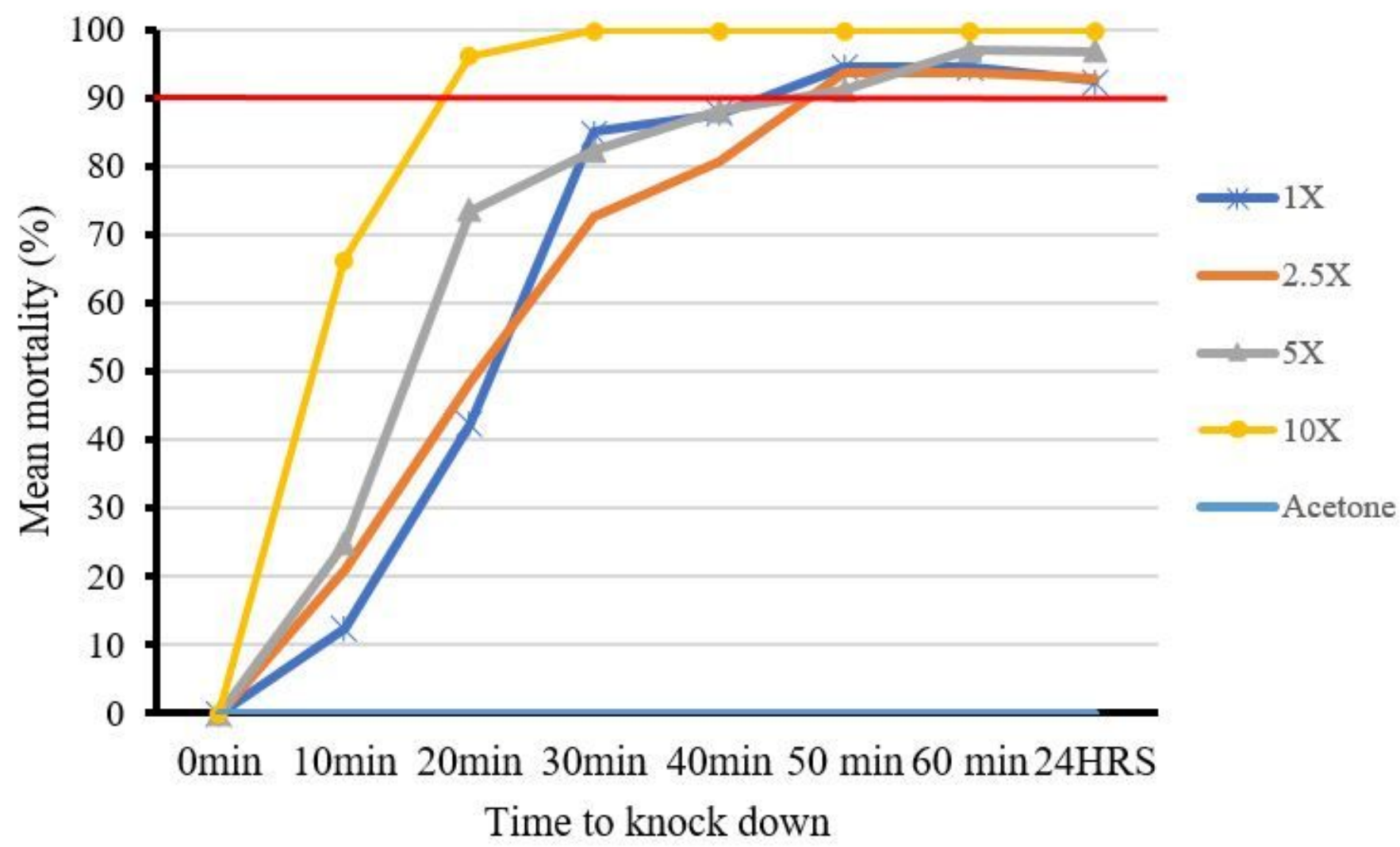

Figure 3

Knockdown time and 24-hr mortality rates of wild-caught An. funestus s.l., after exposure to various concentrations of permethrin. The horizontal redline represents the $90 \%$ mortality threshold for confirmation of resistance 


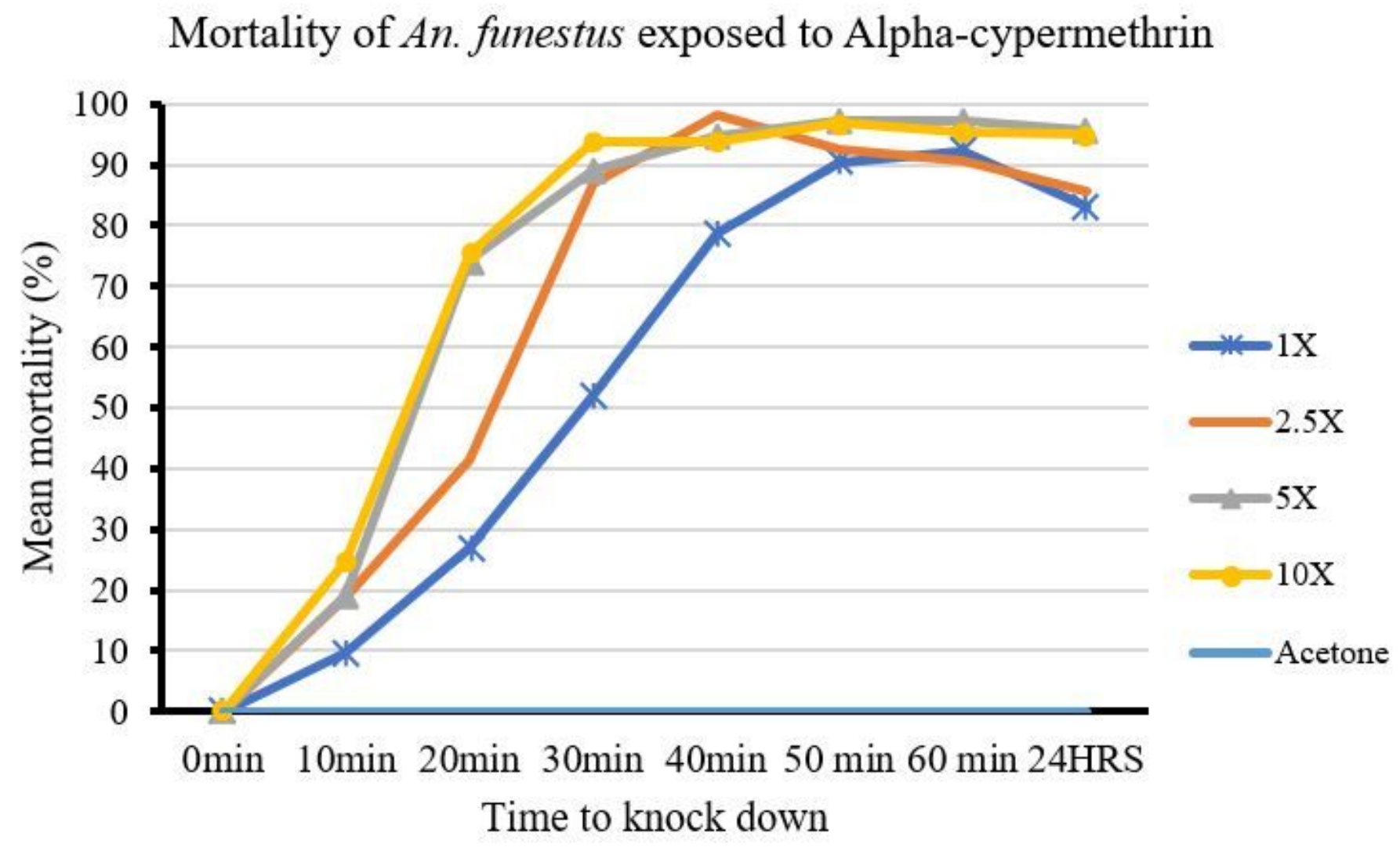

Figure 4

Knockdown time and 24-hr mortality rates of wild-caught An. funestus s.l., after exposure to various concentrations of alpha-cypermethrin. The horizontal redline represents the $90 \%$ mortality threshold for confirmation of resistance 


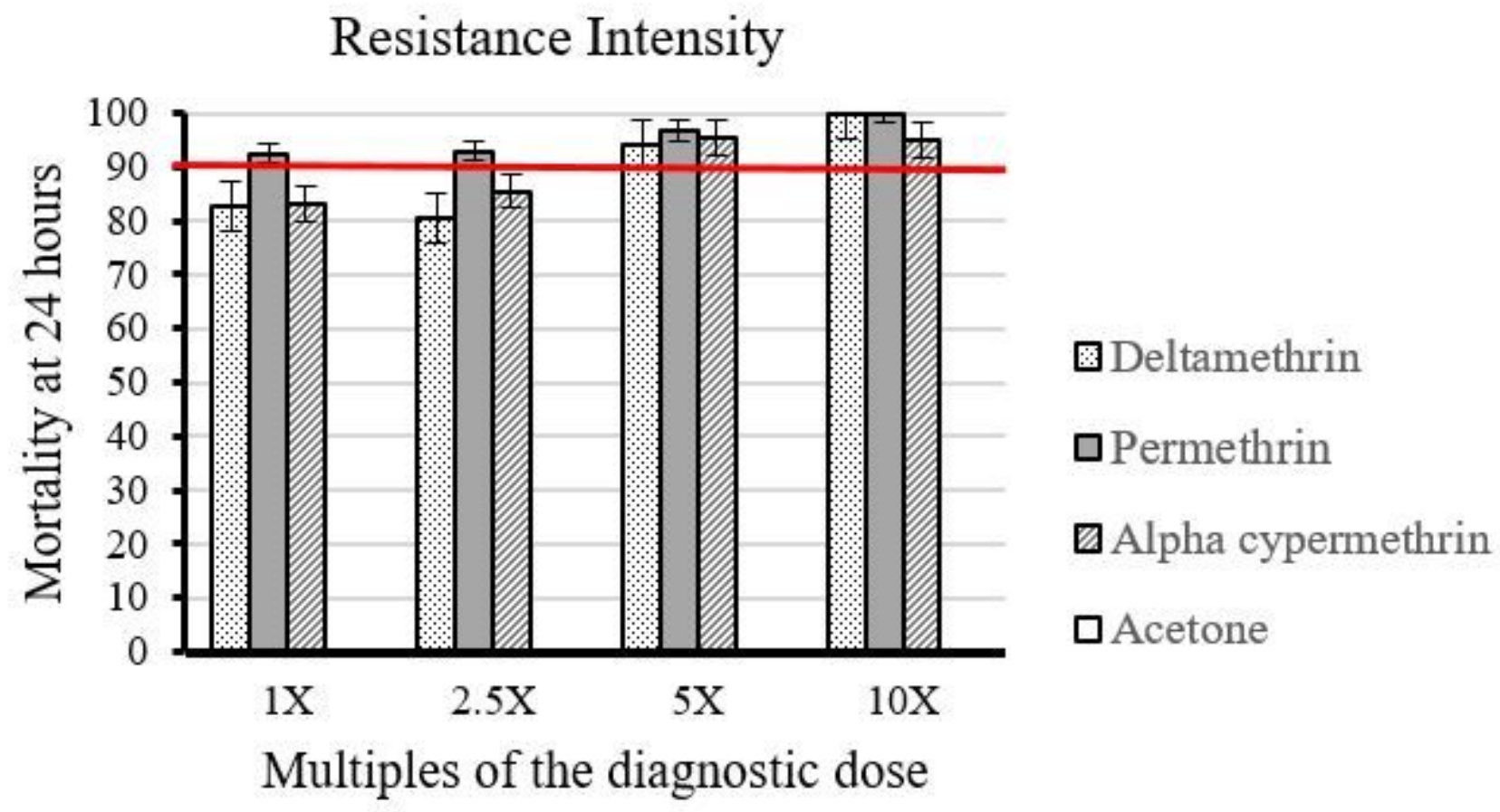

Figure 5

The response of wild An. funestus s./ to different doses of the three pyrethroids is shown. The horizontal redline at $90 \%$ represents the WHO threshold for a resistant population. Error bars represent standard error of mean mortality rates at 24 hours

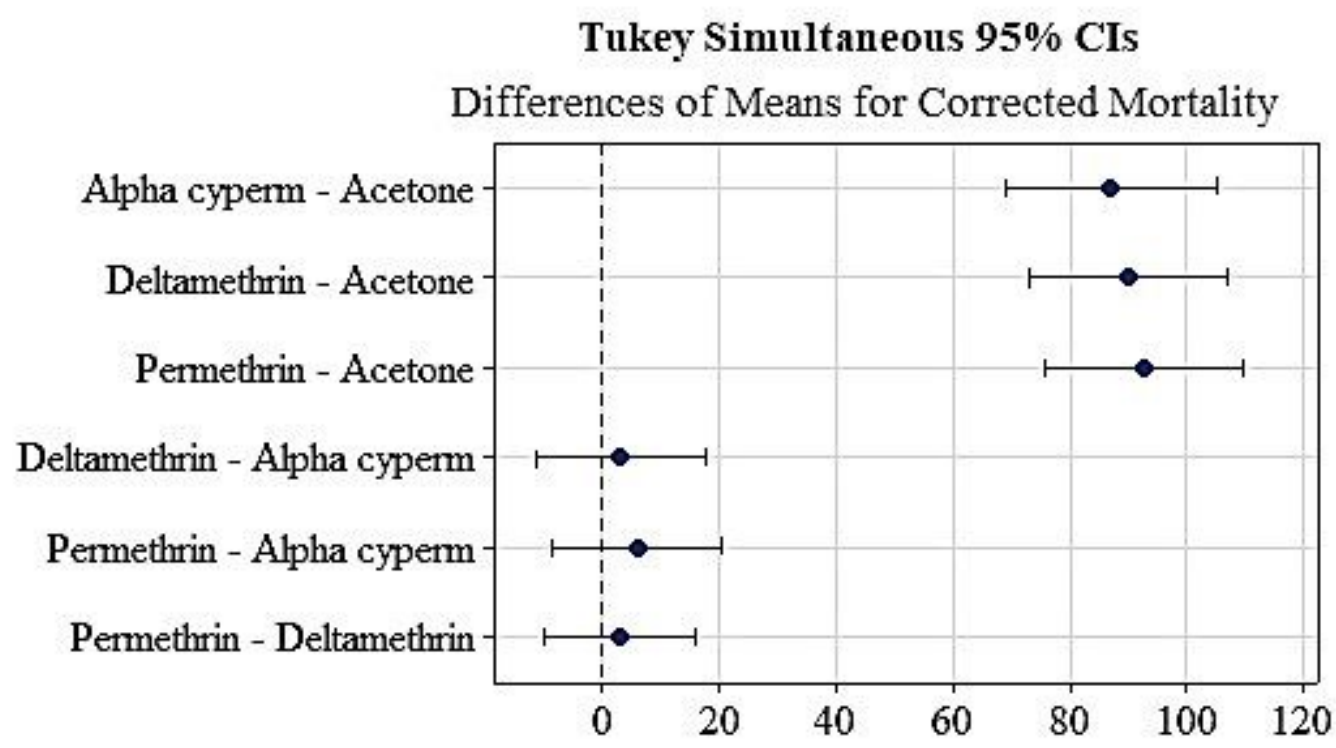

If an interval does not contain zero, the corresponding means are significantly different. 
Figure 6

Pairwise comparisons of mean mortalities for deltamethrin, permethrin, alpha-cypermethrin and acetone negative control

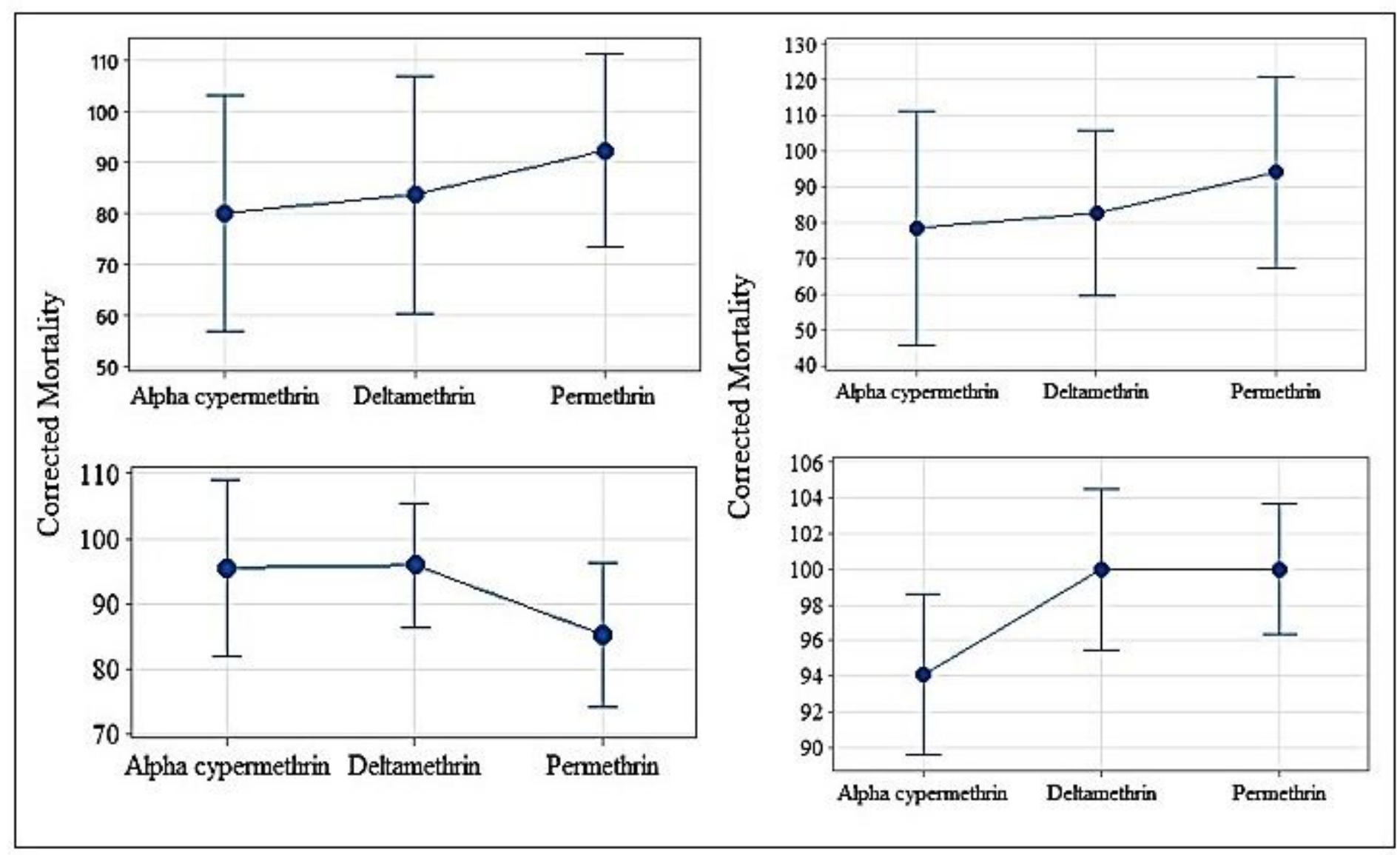

Figure 7

Interval plots for adjusted (corrected) mortality versus dose, for the three pyrethroid insecticides

\section{Supplementary Files}

This is a list of supplementary files associated with this preprint. Click to download.

- Graphicalabstract.pdf 